\title{
Factors associated with technical difficulty of endoscopic submucosal dissection for early gastric cancer that met the expanded indication criteria: post hoc analysis of a multi-institutional prospective confirmatory trial (JCOG0607)
}

\author{
Tomonori Yano ${ }^{1} \cdot$ Noriaki Hasuike ${ }^{2} \cdot$ Hiroyuki Ono ${ }^{3} \cdot$ Narikazu Boku $^{4} \cdot$ Gakuto Ogawa $^{5} \cdot$ Tomohiro Kadota $^{1,5}$. \\ Ichiro Oda ${ }^{6} \cdot$ Hisashi Doyama $^{7}$. Shinichiro Hori ${ }^{8} \cdot$ Hiroyasu lishi $^{9} \cdot$ Akiko Takahashi $^{10} \cdot$ Kohei Takizawa $^{3}$. \\ Manabu Muto ${ }^{11}$
}

Received: 2 May 2019 / Accepted: 8 July 2019 / Published online: 18 July 2019

(c) The International Gastric Cancer Association and The Japanese Gastric Cancer Association 2019

\begin{abstract}
Background There are few reports on the technical difficulty of gastric endoscopic submucosal dissection (ESD). The aim of this study was to investigate the factors associated with the technical difficulty of ESD for early gastric cancer (EGC) using the data from the multicenter non-randomized confirmatory trial of expanded indication criteria of ESD (JCOG0607). Methods The major inclusion criteria were as follows: (1) histologically proven intestinal-type adenocarcinoma; (2) cT1aNOM0; (3) lesion without finding of ulcer (UL-negative) with $>2 \mathrm{~cm}$ in size, or UL-positive with $\leq 3 \mathrm{~cm}$; (4) age 20-75 years. The difficult case was defined as ESD taking $\geq 120 \mathrm{~min}$, piecemeal resection, and/or developing perforation during procedure.

Results Between June 2007 and October 2010, 470 patients were enrolled from 29 institutions. Median procedure time was 79 (range 14-462) min, and it was $\geq 120 \mathrm{~min}$ in 127 patients. Twelve patients developed perforation during ESD, and the procedure time was $\geq 120 \mathrm{~min}$ in 9 of them. Therefore, 130 patients (27.7\%) were identified as difficult cases. Multivariable analysis showed that UL-negative with $>5 \mathrm{~cm}$ (vs. UL-negative with $\leq 3 \mathrm{~cm}$, odds ratio, 24.993; 95\% CI 6.130-101.897, $p<0.0001$ ) had the largest odds ratio and followed by UL-negative with 3-5 cm upper or middle portion of stomach and age $\leq 60$ years were significantly associated with difficulty.

Conclusions UL-negative lesion with $>3 \mathrm{~cm}$, upper or middle portion of stomach and age $\leq 60$ years were independent factors associated with technical difficulty of ESD for EGC. Trial registered number was UMIN000000737.
\end{abstract}

Keywords Early gastric cancer $\cdot$ Endoscopic submucosal dissection $\cdot$ Technical difficulty

Tomonori Yano

toyano@east.ncc.go.jp

1 Department of Gastroenterology and Endoscopy, National Cancer Center Hospital East, 6-5-1, Kashiwanoha, Kashiwa 277-8577, Japan

2 Hasuike Clinic, Kobe, Japan

3 Division of Endoscopy, Shizuoka Cancer Center, Shizuoka, Japan

4 Department of Gastrointestinal Medical Oncology, National Cancer Center Hospital, Tokyo, Japan

5 JCOG Data Center/Operations Office, National Cancer Center Hospital, Tokyo, Japan
6 Endoscopy Division, National Cancer Center Hospital, Tokyo, Japan

7 Department of Gastroenterology, Ishikawa Prefectural Central Hospital, Kanazawa, Japan

8 Department of Gastroenterology, National Hospital Organization Shikoku Cancer Center, Matsuyama, Japan

9 Department of Gastrointestinal Oncology, Osaka International Cancer Institute, Osaka, Japan

10 Department of Endoscopy, Saku Central Hospital Advanced Care Center, Saku, Japan

11 Department of Therapeutic Oncology, Kyoto University Graduate School of Medicine, Kyoto, Japan 


\section{Introduction}

While gastric cancer remains one of the leading causes of cancer death, mortality reduction through early detection with nation-wide endoscopic screening has been successful mainly in Japan and Korea $[1,2]$. Small intramucosal gastric cancer can be easily treated by endoscopic mucosal resection without lymph node dissection. However, in lesions with large size or with ulceration, it is technically difficult to perform endoscopic resection. Endoscopic submucosal dissection (ESD) is now widely indicated for early gastric cancer (EGC) and enables the removal of whole lesions in en bloc fashion regardless of its size and concomitant ulceration (UL-positive) [3].

We previously reported that, in the clinical trial (JCOG0607) of ESD for expanded indication, UL-negative tumor with $>2 \mathrm{~cm}$ in size or UL-positive tumor with $\leq 3 \mathrm{~cm}$ in size, showed equivalent survival to those that underwent surgery with lymph node dissection [4]. Based on these results, the indication was expanded to T1a intestinal type gastric adenocarcinoma with UL-negative $>2 \mathrm{~cm}$ size or UL-positive $\leq 3 \mathrm{~cm}$ size in the clinical guidelines published by Japanese Gastric Cancer Association in 2018.

Clinically, expanded indication might raise the problem of longer procedure time to complete. For example, procedure time longer than 120 min in ESD for EGC was reported as a risk factor of aspiration pneumonia [5]. In addition, perforation is also related with longer procedure time, less completion of ESD. While longer procedure time might be caused by technical difficulty, there were few reports on technical difficulty of ESD for expanded indication of EGC $[6,7]$. As for the technical aspects of the JCOG0607 trial, most of the lesions (91.7\%: 431/470) could be completely resected in en bloc fashion, and the median procedure time was $79 \mathrm{~min}$. However, the procedure time of ESD widely ranged from 14 to 462 min [4].

The aim of this supplementary study was to explore the factors associated with the technical difficulty of ESD for EGC, which met the expanded indication criteria using the data from JCOG0607.

\section{Methods}

This post hoc analysis used the data collected in a multiinstitutional prospective confirmatory trial of ESD for EGC which met the expanded indication criteria (JCOG0607). The primary endpoint of JCOG0607 was the 5 -year overall survival rate after ESD with an intention to treat analysis. JCOG0607 complied with the Declaration of Helsinki requirements, which was approved by the institutional review board of all participating hospitals, and registered with the University Hospital Medical Information Network Clinical Trials Registry (UMIN000000737). The informed consent about the secondary use of the data was obtained from the enrolled patients at the registration to JCOG0607.

\section{Inclusion criteria of JCOG0607 and the definition of difficult case in this study}

The key indication criteria of JCOG0607 were as follows: (1) primary and single histologically proven intestinal-type adenocarcinoma of the stomach, (2) endoscopically diagnosed mucosal tumor and absence of lymph node and distant metastasis (cT1aN0M0) in computed tomography (CT) of abdomen, (3) UL-negative tumor with $>2 \mathrm{~cm}$ in size or ULpositive tumor with $\leq 3 \mathrm{~cm}$ in size, (4) age of 20-75 years, (5) Eastern Cooperative Oncology Group performance status of 0 or 1, (6) no prior gastrectomy and no reconstructive surgery using the stomach after resection of esophageal cancer, (7) no prior chemotherapy or radiation therapy for any other malignancies, (8) high possibility of en bloc resection with ESD, and (9) provision of written informed consent [4]. The difficult case was defined as ESD taking 120 min or longer, and/or piecemeal resection and/or developing perforation during the procedure. In JCOG0607, the procedure time was defined from the start of marking to the end of resection, and the perforation was diagnosed as follows: (1) mesenteric fat was observed with endoscopy during ESD or, (2) free air was detected with X-ray or CT of abdomen. The easy case was defined as ESD taking less than 120 min with en bloc resection without perforation during procedure.

\section{ESD procedure and trial profile of JCOG0607}

In JCOG0607, all ESD procedures were performed by certified endoscopists as operator or supervisor, who had experienced 100 cases of gastric ESD or more. ESD was performed in a manner previously reported $[8,9]$. The device for ESD depended on the operators' choices such as IT-knife ${ }^{\circledR}$, Hook knife ${ }^{\circledR}$, Flush knife ${ }^{\circledR}$, and others. After the completion of ESD, all resected materials were evaluated by experienced pathologists at each participating institution.

\section{Statistical analysis}

All data including procedure time were collected prospectively. Univariable and multivariable analyses of the proportion of difficult cases were performed with following categorized variables: age, sex, location (U: upper, M: middle, L: lower), circumference location (on greater curvature, non-spreading to greater curvature), macroscopic 
type (protruded, depressed, mixed), tumor size and UL finding, device, institution, and registration periods. Institution was classified into three groups depending on the numbers of registered patients, and the whole registration period was divided into three periods (first $1 / 3$ period: June 2007-June 2008, middle $1 / 3$ period: June 2008-June 2009, last $1 / 3$ period: June $2009-O c t 2010$ ). As for age, the proportion of difficult cases was compared among 4 categories of age ( $\leq 60$ years, $61-65$ years, $66-70$ years, $>71$ years) to set the cut-off for categorizing to be used in univariable and multivariable analyses. Because of the indication criteria of JCOG0607, all lesions $>3 \mathrm{~cm}(n=111)$ were UL-negative and all lesions with UL-positive were $\leq 3 \mathrm{~cm}$ $(n=207)$, it is difficult to analyze size and UL finding as each variables in multivariable analysis due to high correlation. Therefore, we used four categories combining size and UL finding (UL-negative and $\leq 3 \mathrm{~cm}$, UL-negative and $3-5 \mathrm{~cm}$, UL-negative and $>5 \mathrm{~cm}$, UL-positive) in this study. As reference, univariable analysis using tumor size as continuous variable was performed, and the proportion of difficult case was compared among the five categories of tumor size ( $\leq 2 \mathrm{~cm}, 2-2.5 \mathrm{~cm}, 2.5-3 \mathrm{~cm}, 3-5 \mathrm{~cm},>5 \mathrm{~cm}$ ).

Odds ratios were estimated using logistic regression model using the above-mentioned variables. Fisher's exact test was also used for univariable analyses. All $p$ values were two-sided. Statistical analyses were performed by a biostatistician of the JCOG Data Center using SAS version 9.4 .

\section{Results}

Between June 2007 and October 2010, 470 patients were enrolled and all of them underwent ESD in JCOG0607. This post hoc analysis included all 470 patients. As for cutting devices for ESD, IT-knife or IT-knife 2 were predominant (82.3\%: 387/470), and approximately $75 \%$ of the patients (355/470) enrolled were from the top one-third active institutions. ESD was stopped during the procedure in one case because of perforation. The procedure time was $120 \mathrm{~min}$ or longer in 127 patients, and 9 of them were complicated even with perforation during ESD. Moreover, 3 cases developed perforation with their procedure time less than $120 \mathrm{~min}$. In total, $130(27.7 \%)$ cases were classified as difficult cases, whereas the other 340 cases were easy cases (Fig. 1). All patients' and lesions' characteristics in both difficult and easy groups are summarized in Table 1 . The details related to ESD procedure are summarized in Table 2. The median procedure time was 61.5 (range 14-119) min in easy cases and 157.5 (range 48-462) $\mathrm{min}$ in the difficult cases. The histogram of the procedure time in each group was presented in Fig. 2.

\section{Enrollment and ESD $(n=470)$}

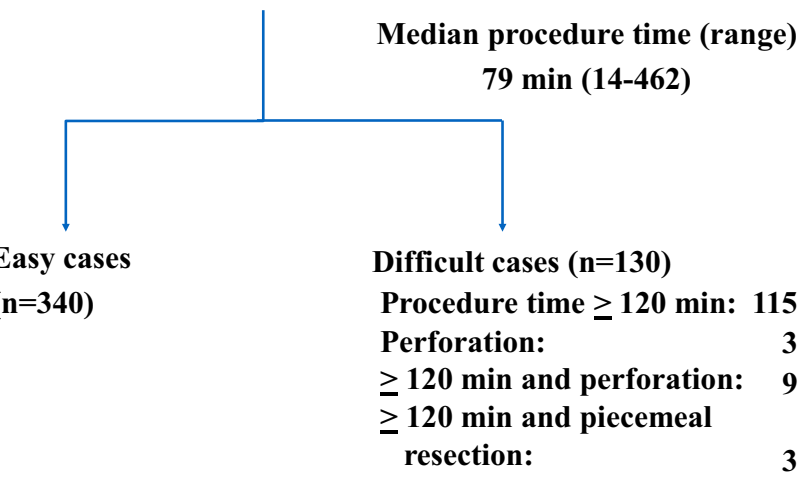

Fig. 1 The patients' flow diagram

Table 1 Patients' and lesions' characteristics

\begin{tabular}{ll}
\hline & Total $(n=470)$ \\
\hline Median age (y-o, range) & $65(40-75)$ \\
Gender & \\
M & 385 \\
F & 85 \\
Location & \\
U & 71 \\
M & 255 \\
L & 144 \\
Tumor size (cm) & \\
Median & 2.5 \\
Range & $0.5-13$ \\
Macroscopic type & \\
Protruded & 155 \\
Depressed & 258 \\
Mixed & 57 \\
Ulceration or scar & \\
Presence $(U L+)$ & 207 \\
Absence $(U L-)$ & 263 \\
Tumor size and finding of UL & \\
UL $(-)$ and $\leq 3 \mathrm{~cm}$ & 152 \\
UL $(-)$ and $>3 \mathrm{~cm}$ & 111 \\
UL (+) $(<3 \mathrm{~cm})$ & 207 \\
\hline
\end{tabular}

\section{Univariable analysis of factors associated with technical difficulty}

The results of the univariable analyses for the technical difficulty are presented in Table 3 . As for age, the proportion of difficult cases was $33.3 \%$ (47/141) in patients aged $\leq 60$ years, $25.5 \%$ (28/110) in those aged 61-65 years, $24.0 \%(29 / 121)$ in those aged $66-70$ years, and $26.5 \%$ (26/98) in those aged $>71$ years. The proportions of difficult cases were consistent in patients $>60$ years while it was 
Table 2 Details of ESD procedure

\begin{tabular}{lllll}
\hline & $\begin{array}{l}\text { Easy cases } \\
(n=340)\end{array}$ & $\begin{array}{l}\text { Difficult } \\
\text { cases } \\
(n=130)\end{array}$ & Total (470) & $\begin{array}{l}\text { \% of } \\
\text { difficult } \\
\text { cases }\end{array}$ \\
\hline Median procedure time (min) & 61.5 & 157.5 & 79 & \\
Range (min) & $14-119$ & $48-462$ & $14-462$ & \\
Main device using in ESD (IT-knife/non IT-knife) & & & & 26.9 \\
IT-knife or IT-knife 2 & 283 & 104 & 387 & 31.3 \\
Others (Hook knife, flush knife, flex knife, etc.) & 57 & 26 & 83 & 27.6 \\
Institution (number of enrollment) & & & & 28.0 \\
Top 1/3 & 257 & 98 & 355 & 27.2 \\
Middle 1/3 & 67 & 26 & 93 & 31.4 \\
Lower 1/3 & 16 & 6 & 22 & 24.3 \\
Registration period & & & & 25.9 \\
First 1/3 period (2007/6/8-2008/6/7) & 107 & 49 & 156 & 144 \\
Middle 1/3 period (2008/6/8-2009/6/7) & 109 & 35 & 170 & \\
Last 1/3 period (2009/6/8-2010/10/4) & 126 & 44 & & \\
\hline
\end{tabular}

Fig. 2 The histogram of procedure time in each groups

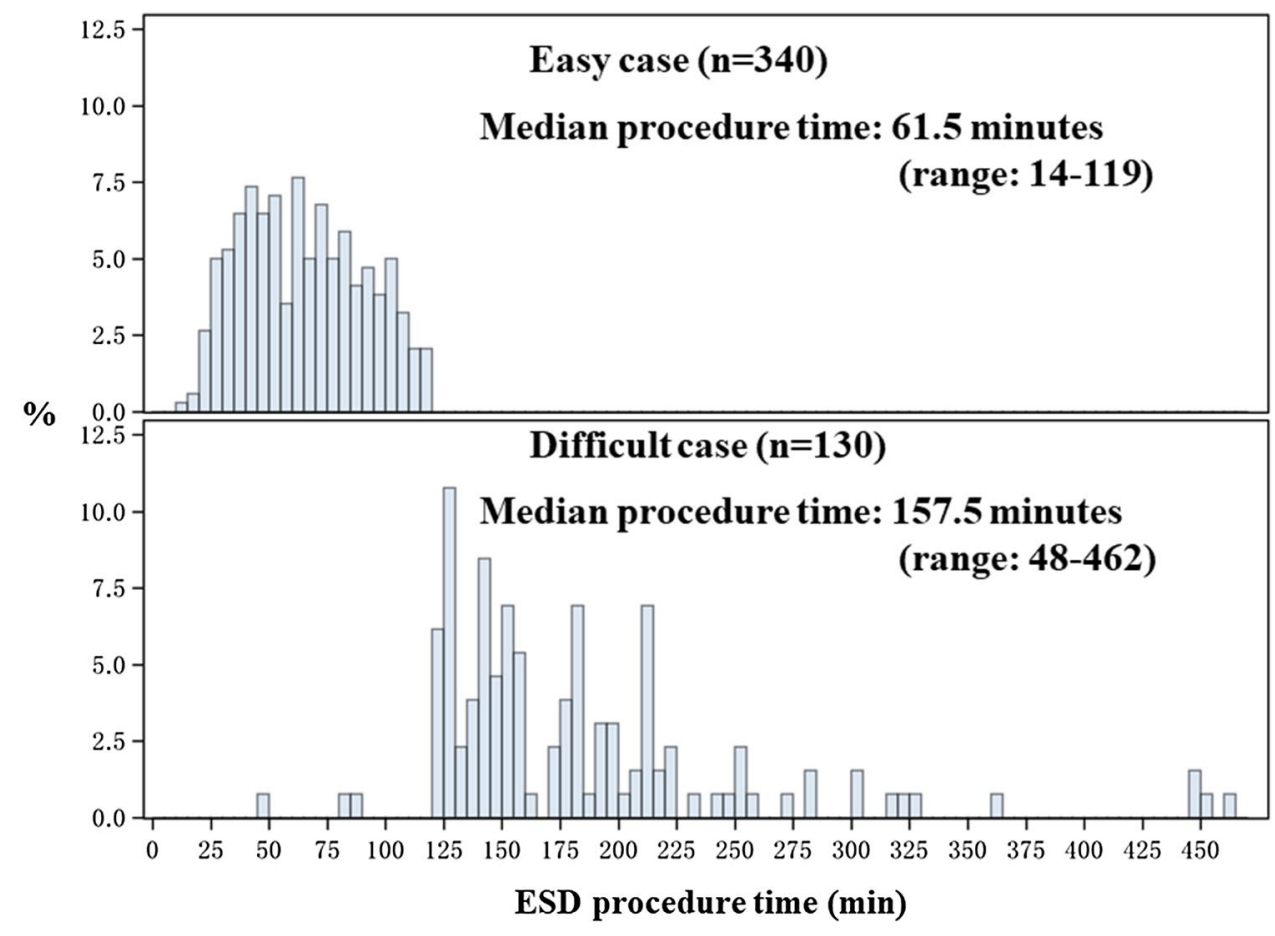

relatively higher in patients $\leq 60$ years old. In univariable analysis using categorized variable for age $\leq 60$ years (odds ratio $1.482 ; 95 \%$ CI $0.964-2.277 ; p=0.0727$ ), location of upper $1 / 3$ in stomach (odds ratio 2.433 ; 95\% CI 1.297-4.564; $p=0.0056$ ), UL-negative tumor of $3-5 \mathrm{~cm}$ size (odds ratio 3.444; 95\% CI 1.938-6.122, $p<0.0001)$, and UL-negative and $>5 \mathrm{~cm}$ size (odds ratio 17.711; 95\% CI 4.685-66.959, $p<0.0001)$ were significantly associated with technical difficulty. As reference, univariable analysis of tumor size $(\mathrm{cm})$ as the continuous variable was performed and an odds ratio was 1.364 (95\% CI 1.240-1.501, $p<0.0001)$, the proportion of the difficult case was related to the tumor size: $16.7 \%$ $(23 / 138)$ in $\leq 2 \mathrm{~cm}, 21.3 \%(26 / 122)$ in $>2 \mathrm{~cm}$ and $\leq 2.5 \mathrm{~cm}$, $27.3 \%(27 / 99)$ in $>2.5 \mathrm{~cm}$ and $\leq 3 \mathrm{~cm}, 43.8 \%(42 / 96)$ in $>3 \mathrm{~cm}$ and $\leq 5 \mathrm{~cm}$, and $80.0 \%(12 / 15)$ in $>5 \mathrm{~cm}$.

\section{Multivariable analysis of factors associated with technical difficulty}

In multivariable analysis, age $\leq 60$ years (odds ratio 1.755 ; 95\% CI 1.101-2.799; $p=0.0181$ ), location of upper $1 / 3$ (odds ratio $3.192 ; 95 \%$ CI $1.579-6.452 ; p=0.0012$ ) and 
Table 3 The univariable and multivariable analyses for the technical difficulty

\begin{tabular}{|c|c|c|c|c|c|c|c|c|c|}
\hline & \multirow{2}{*}{\multicolumn{2}{|c|}{$\begin{array}{l}\text { Proportion of dif- } \\
\text { ficult case }\end{array}$}} & \multirow[t]{2}{*}{$p$ value } & \multicolumn{3}{|c|}{ Univariable analysis } & \multicolumn{3}{|c|}{ Multivariable analysis } \\
\hline & & & & Odds ratio & $(95 \% \mathrm{CI})$ & $p$ value & Odds ratio & $(95 \% \mathrm{CI})$ & $p$ value \\
\hline \multicolumn{10}{|l|}{ Age (years) } \\
\hline$>61$ & $83 / 329$ & $25.2 \%$ & & 1 & & 0.0727 & 1 & & 0.0181 \\
\hline$\leq 60$ & $47 / 141$ & $33.3 \%$ & 0.0911 & 1.482 & $0.964-2.277$ & & 1.755 & $1.101-2.799$ & \\
\hline \multicolumn{10}{|l|}{ Gender } \\
\hline Female & $19 / 85$ & $22.4 \%$ & & 1 & & 0.2285 & 1 & & \\
\hline Male & $111 / 385$ & $28.8 \%$ & 0.2837 & 1.407 & $0.807-2.453$ & & 1.542 & $0.835-2.848$ & 0.1667 \\
\hline \multicolumn{10}{|l|}{ Location } \\
\hline $\mathrm{L}$ & $29 / 144$ & $20.1 \%$ & & 1 & & & 1 & & \\
\hline M & $74 / 255$ & $29.0 \%$ & & 1.621 & $0.994-2.643$ & 0.0527 & 1.764 & $1.017-3.061$ & 0.0433 \\
\hline $\mathrm{U}$ & $27 / 71$ & $38.0 \%$ & 0.0171 & 2.433 & $1.297-4.564$ & 0.0056 & 3.192 & $1.579-6.452$ & 0.0012 \\
\hline \multicolumn{10}{|l|}{ Circumferential location } \\
\hline $\begin{array}{l}\text { Non-spreading to } \\
\text { greater curvature }\end{array}$ & $117 / 406$ & $28.8 \%$ & & 1 & & & 1 & & \\
\hline On greater curvature & $13 / 64$ & $20.3 \%$ & 0.1778 & 0.630 & $0.330-1.201$ & 0.1606 & 0.703 & $0.346-1.426$ & 0.3284 \\
\hline \multicolumn{10}{|l|}{ Macroscopic type } \\
\hline Protruded & $54 / 155$ & $34.8 \%$ & & 1 & & & 1 & & \\
\hline Depressed & $62 / 258$ & $24.0 \%$ & & 0.592 & $0.382-0.916$ & 0.0185 & 0.669 & $0.369-1.211$ & 0.1838 \\
\hline Mixed & $14 / 57$ & $24.6 \%$ & 0.0553 & 0.609 & $0.306-1.211$ & 0.1574 & 1.004 & $0.458-2.204$ & 0.9915 \\
\hline \multicolumn{10}{|l|}{ Tumor size and UL } \\
\hline $\mathrm{UL}(-)$ and $\leq 3 \mathrm{~cm}$ & $28 / 152$ & $18.4 \%$ & & 1 & & & 1 & & \\
\hline $\mathrm{UL}(-)$ and $3-5 \mathrm{~cm}$ & $42 / 96$ & $43.8 \%$ & & 3.444 & $1.938-6.122$ & $<0.0001$ & 3.765 & $2.052-6.908$ & $<0.0001$ \\
\hline $\mathrm{UL}(-)$ and $>5 \mathrm{~cm}$ & $12 / 15$ & $80.0 \%$ & & 17.711 & $4.685-66.959$ & $<0.0001$ & 24.993 & $6.130-101.897$ & $<0.0001$ \\
\hline $\mathrm{UL}(+)(\leq 3 \mathrm{~cm})$ & $48 / 207$ & $23.2 \%$ & $<0.0001$ & 1.337 & $0.793-2.253$ & 0.2755 & 1.683 & $0.916-3.091$ & 0.0933 \\
\hline \multicolumn{10}{|l|}{ Device } \\
\hline IT knife or IT knife 2 & $104 / 387$ & $26.9 \%$ & & 1 & & & 1 & & \\
\hline Others & $26 / 83$ & $31.3 \%$ & 0.4191 & 1.241 & $0.741-2.078$ & 0.4112 & 1.256 & $0.669-2.360$ & 0.4779 \\
\hline \multicolumn{10}{|c|}{ Institution (number of enrollment) } \\
\hline Top $1 / 3$ & $98 / 355$ & $27.6 \%$ & & 1 & & & 1 & & \\
\hline Middle 1/3 & $26 / 93$ & $28.0 \%$ & & 1.018 & $0.612-1.693$ & 0.9461 & 1.131 & $0.620-2.066$ & 0.6880 \\
\hline Lower $1 / 3$ & $6 / 22$ & $27.3 \%$ & 1.0000 & 0.983 & $0.374-2.586$ & 0.9731 & 1.003 & $0.350-2.878$ & 0.9951 \\
\hline \multicolumn{10}{|l|}{ Registration period } \\
\hline First $1 / 3$ period & $49 / 156$ & $31.4 \%$ & & 1 & & & & & \\
\hline Middle $1 / 3$ period & $35 / 144$ & $24.3 \%$ & & 0.701 & $0.421-1.167$ & 0.1718 & 0.706 & $0.406-1.229$ & 0.2183 \\
\hline Last $1 / 3$ period & $46 / 170$ & $27.1 \%$ & 0.3804 & 0.810 & $0.502-1.307$ & 0.3881 & 0.856 & $0.507-1.446$ & 0.5607 \\
\hline
\end{tabular}

middle $1 / 3$ of the stomach (odds ratio $1.764 ; 95 \%$ CI 1.017-3.061), UL-negative tumor of 3-5 cm size (odds ratio 3.765 ; 95\% CI $2.052-6.908 ; p<0.0001)$ and ULnegative tumor of $>5 \mathrm{~cm}$ size (odds ratio 24.993 ; $95 \%$ CI $6.130-101.897, p<0.0001)$ were significantly associated with technical difficulty.

\section{Discussion}

This is the first report that evaluated the technical difficulty of gastric ESD for expanded indication criteria using data of a multi-institutional prospective study. Although this supplementary study was not pre-planned, all data used in this post hoc analysis had been already collected prospectively, including the ESD procedure time and adverse events. This study demonstrated that the UL-negative tumor with $>5 \mathrm{~cm}$ size showed the largest odds ratio, followed by UL-negative tumor of $3-5 \mathrm{~cm}$ size, upper or middle $1 / 3$ location and age $\leq 60$ years, as a significant risk factor of difficult ESD taking $\geq 120$ min or complicated with perforation.

Tumors with large size or located in the upper part of the stomach were known factors associated with technical difficulty, as described in previous reports $[6,7,10]$. The procedure of ESD generally consists of the following three major processes; (1) cutting the surrounding of the lesion, 
(2) dissection of submucosal layer beyond the lesion, and (3) hemostasis for intraoperative bleeding. During the process of dissection of the submucosal layer, minor and/or major bleeding make complicated the procedure in some cases. In other words, dissection of the submucosal layer and hemostasis were procedures performed alternately and repeatedly. Management of bleeding includes the steps of searching the bleeding spot and stopping bleeding, which results in time consuming. The risk of bleeding is getting higher with the larger size of dissected area. Thus, it is reasonable that lesion size is the most important risk factor of difficulty in ESD. Goto et al. reported that both the presence of ulcerative findings and tumor size larger than $2 \mathrm{~cm}$ were the significant predictors for procedure time longer than 120 min [6]. Nagata et al. also described that ulcerative change was related to longer procedure time [7]. In this study, ULpositive was not a significant factor for technical difficulty by multivariable analysis, probably because all UL-positive lesions were less than $3 \mathrm{~cm}$ in size. It is considered that the recent ESD technique using new devices might conquer the difficulty due to the presence of ulceration in relatively small $(<3 \mathrm{~cm})$ lesions.

Similar to this study, there have been some reports that EGC in located in the upper or middle part of the stomach required a significant longer ESD procedure time than those located in the lower stomach $[6,10]$. It is considered that technical difficulty due to location cannot be resolved only by improvement of cutting device. Moreover, tangential approach is required when dissecting the submucosal layer beneath the lesion especially on greater curvature in upper stomach. Recently, some investigators reported the innovative methods using traction devices when dissecting the submucosal layer to resolve the technical difficulty in gastric ESD [11-14]. Yoshida et al. conducted a multi-center randomized control trial of conventional ESD versus dental floss clip traction assisted ESD (DFC-ESD) for early gastric neoplasm [15]. While DFC-ESD failed to significantly reduce the procedure time compared with the conventional method in the analysis of all enrolled lesions, DFC traction tended to shorten the procedure time at the area of greater curvature of the middle or upper part of the stomach in a subset analysis. As the traction device was generalized after the study period, most of the cases were performed without those tools. These new traction devices, which are used recently, may solve the technical difficulty related to the location of lesion in gastric ESD.

Age of $\leq 60$ years was one of the risk factors associated with technical difficulty in this study. There are few reports comparing the procedure time between elderly and young patients in gastric ESD. Iwai et al. reported the retrospective comparative study between elderly ( $\geq 80$ years) and non-elderly ( $<80$ years) patients to assess short- and longterm outcome of gastric ESD [16]. There was no significant difference in the procedure time and incidence of perforation between the two groups; however, it might be difficult to refer the results of their study into our study. Because it was the retrospective analysis of their daily practical data, the lesions' characteristics of each group were different. Meanwhile, somatotype and mucosal condition might explain the reason for the differences in the findings between young and elderly patients. High body mass index (BMI) was reported as significantly associated with long operation time in gastrectomy due to thick abdominal wall and massive adipose tissue in clinical setting [17]. Thick gastric wall or increased adipose tissue in the submucosal layer might be associated with long procedure time in ESD as well as gastrectomy. In general, gastric atrophy increased with age especially in Helicobacter pylori-infected stomach [18], and decreased gastric mucosal blood flow with aging as reported in experimental models [19]. The difference in the degree of gastric atrophy or mucosal blood flow with age can affect the prevalence of technical difficulty. However, it cannot conclude that these factors affect the difficulty of ESD because information of patients' physics (e.g. BMI), degree of gastric atrophy, and $H$. pylori infection status were not collected in JCOG0607. This is one of the major limitations of this study, and further investigation is necessary to elucidate this issue.

Moreover, the second limitation was that there was no control group, including small $(<2 \mathrm{~cm})$ and UL-negative lesions because of the indication criteria of JCOG0607. Therefore, fair comparison of the technical difficulty between UL-positive and -negative cases of same size could not be performed. Finally, information about the specific time required for each step of the procedure is lacking. These data may contribute to the clear identification of risk factors and the developing of new devices for ESD. To know the accurate time allotted for each step based on the technical aspects will also be when training young endoscopists and for the prediction of procedure time. Furthermore, it may have an educational benefit in the case selection for novice operator or in providing information about the operation time for patients and their family members before the procedure.

In conclusion, UL negative tumor $>3 \mathrm{~cm}$ size, location in the upper or middle part of the stomach, and age of 60 years or younger were the independent risk factors associated with technical difficulty in ESD for EGC, which met the expanded indication criteria. While traction devices including dental floss clip may be supportive to conquer the factors related to lesion's location in technical difficulty, we should continue to make an effort to innovate other devices or methods to reduce adverse events related to long procedure time of gastric ESD.

Acknowledgements This post hoc analysis was used collected data of JCOG0607 that was supported by Grants-in-Aid for Cancer Research 
(17S-3, 17S-5, 20S-3, 20S-6, 23-A-16, 23-A-19, 26-A-4, 29-A-3) and Health and Labour Sciences Research Grants for Clinical Cancer Research (17-12) (20-15) from the Ministry of Health, Labour and Welfare, Japan. Participating institutions: Gastrointestinal Endoscopy Study Group of the Japan Clinical Oncology Group (JCOG); Hokkaido University Hospital; Federation of National Public Service Personal Mutual Aid Associations, Tonan Hospital; Tokyo Metropolitan Cancer and Infectious Diseases Center Komagome Hospital; Asahi General Hospital; Ishikawa Prefectural Central Hospital, Saku Central Hospital Advanced Care Center; Shizuoka Cancer Center; Aichi Cancer Center Hospital; Aichi Cancer Center, Aichi Hospital; Kyoto University Hospital; Osaka Prefectural Hospital Organization; Osaka International Cancer Institute; Osaka City General Hospital; Kobe University Graduate School of Medicine; Hyogo Cancer Center; National Hospital Organization Shikoku Cancer Center; Kochi Health Sciences Center; Tochigi Cancer Center; Yamagata Prefectural Central Hospital; Ibaraki Central Hospital and Cancer Center; Showa University School of Medicine; Cancer Institute Hospital of Japanese Foundation for Cancer Research; Toranomon Hospital; Yokohama City University Medical Center; Kanagawa Cancer Center; Kitasato University School of Medicine; National Cancer Center Hospital East; National Cancer Center Hospital; Iwate Prefectural Central Hospital.

\section{Compliance with ethical standards}

Conflict of interest All authors declare no conflict of interest related to this article.

\section{References}

1. Choi KS, Jun JK, Suh M, et al. Effect of endoscopy screening on stage at early gastric cancer diagnosis: results of the National Cancer Screening Programme in Korea. Br J Cancer. 2015;112:608-12.

2. Hamashima C, Ogoshi K, Okamoto M, et al. A communitybased, case-control study evaluating mortality reduction from gastric cancer by endoscopic screening in Japan. PLoS ONE. 2013;8(11):e79088. https://doi.org/10.1371/journal.pone.00790 88.

3. Ono H, Kondo H, Gotoda T, et al. Endoscopic mucosal resection for treatment for early gastric cancer. Gut. 2001;48:225-9.

4. Hasuike N, Ono H, Boku N, et al. A non-randomized confirmatory trial of an expanded indication for endoscopic submucosal dissection for intestinal-type gastric cancer (cT1a): the Japan Clinical Oncology Group study (JCOG0607). Gastric Cancer. 2018;21(1):114-23. https://doi.org/10.1007/s10120-017-0704-y.

5. Park CH, Kim H, Kang YA, et al. Risk factors and prognosis of pulmonary complications after endoscopic submucosal dissection for gastric neoplasia. Dig Dis Sci. 2013;58(2):540-6. https://doi. org/10.1007/s10620-012-2376-0 (epub 2012 Sep 21).

6. Goto O, Fujishiro M, Kodashima S, et al. Is it possible to predict the procedural time of endoscopic submucosal dissection for early gastric cancer? J Gastroenterol Hepatol. 2009;24(3):379-83.
7. Nagata S, Jin YF, Tomoeda M, et al. Influential factors in procedure time of endoscopic submucosal dissection for gastric cancer with fibrotic change. Dig Endosc. 2011;23(4):296-301.

8. Gotoda T. A large endoscopic resection by endoscopic submucosal dissection procedure for early gastric cancer. Clin Gastroenterol Hepatol. 2005;3:S71-S7373.

9. Gotoda T, Yamamoto H, Soetikno R. Endoscopic submucosal dissection of early gastric cancer. J Gastroenterol. 2006;41:929-42.

10. Chung IK, Lee JH, Lee SH, et al. Therapeutic outcomes in 1000 cases of endoscopic submucosal dissection for early gastric neoplasms: Korean ESD Study Group multicenter study. Gastrointest Endosc. 2009;69(7):1228-355. https://doi.org/10.1016/j. gie.2008.09.027 (epub 2009 Feb 27).

11. Jeon WJ, You IY, Chae HB, Park SM, Youn SJ. A new technique for gastric endoscopic submucosal dissection: peroral tractionassisted endoscopic submucosal dissection. Gastrointest Endosc. 2009;69(1):29-33.

12. Hijikata Y, Ogasawara N, Sasaki M, et al. Endoscopic submucosal dissection with sheath-assisted counter traction for early gastric cancers. Dig Endosc. 2010;22(2):124-8.

13. Aihara H, Kumar N, Ryou M, et al. Facilitating endoscopic submucosal dissection: the suture-pulley method significantly improves procedure time and minimizes technical difficulty compared with conventional technique: an ex vivo study (with video). Gastrointest Endosc. 2014;80(3):495-502.

14. Yoshida M, Takizawa K, Ono H, et al. Efficacy of endoscopic submucosal dissection with dental floss clip traction for gastric epithelial neoplasia: a pilot study (with video). Surg Endosc. 2016;30(7):3100-6.

15. Yoshida M, Takizawa K, Suzuki S, et al. Conventional versus traction-assisted endoscopic submucosal dissection for gastric neoplasms: a multicenter, randomized controlled trial (with video). Gastrointest Endosc. 2018;87(5):1231-40.

16. Iwai $\mathrm{N}$, Dohi $\mathrm{O}$, Naito $\mathrm{Y}$, et al. Impact of the Charlson comorbidity index and prognostic nutritional index on prognosis in patients with early gastric cancer after endoscopic submucosal dissection. Dig Endosc. 2018;30(5):616-23.

17. Feng F, Zheng G, Guo X, et al. Impact of body mass index on surgical outcomes of gastric cancer. BMC Cancer. 2018;18(1):151. https://doi.org/10.1186/s12885-018-4063-9.

18. Sonnenberg A, Genta RM. Changes in the gastric mucosa with aging. Clin Gastroenterol Hepatol. 2015;13(13):2276-81.

19. Tarnawski A, Pai R, Deng X, et al. Aging gastropathy-novel mechanisms: hypoxia, up-regulation of multifunctional phosphatase PTEN, and proapoptotic factors. Gastroenterology. 2007;133(6):1938-47 (epub 2007 Aug 21).

Publisher's Note Springer Nature remains neutral with regard to jurisdictional claims in published maps and institutional affiliations. 